\title{
EGCG suppresses prostate cancer cell growth modulating acetylation of androgen receptor by anti-histone acetyltransferase activity
}

\author{
YOO-HYUN LEE ${ }^{1 *}$, JIEUN KWAK $^{2 *}, \mathrm{HYO}^{2}$ YYOUNG CHOI $^{3}, \mathrm{KYUNG-CHUL} \mathrm{CHOI}^{4}, \mathrm{SUNOH} \mathrm{KIM}^{5}$, \\ JEONGMIN LEE $^{6}$, WOOJIN JUN ${ }^{7}$, HYUN-JIN PARK ${ }^{2}$ and HO-GEUN YOON ${ }^{3}$
}

\begin{abstract}
${ }^{1}$ Department of Food Science and Nutrition, The University of Suwon, Kyunggi-do; ${ }^{2}$ Brain Korea 21 Life Science and Biotechnology, Korea University, Seoul; ${ }^{3}$ Department of Biochemistry and Molecular Biology, Center for Chronic Metabolic Disease Research, College of Medicine, Yonsei University, Seoul; ${ }^{4}$ Department of Medicine, Graduate School, University of Medicine, Seoul; ${ }^{5}$ Jeollanamdo Institute of Natural Resources Research, Jeonnam; ${ }^{6}$ Department of Medical Nutrition, Kyung Hee University, Kyunggi-do; ${ }^{7}$ Department of Food and Nutrition, Chonnam National University, Gwangju, Republic of Korea
\end{abstract}

Received January 19, 2012; Accepted March 16, 2012

DOI: $10.3892 / \mathrm{ijmm} .2012 .966$

\begin{abstract}
Manipulating acetylation status of key gene targets is likely to be crucial for effective cancer therapy. In this study, we utilized green tea catechins, epicatechin (EC), epigallocatechin (EGC) and epigallocatechin-3-gallate (EGCG) to examine the regulation of androgen receptor acetylation in androgen-dependent prostate cancer cells by histone acetyltransferase (HAT) activity. EC, EGC and EGCG induced prostate cancer cell death, suppressed agonist-dependent androgen receptor (AR) activation and AR-regulated gene transcription. These results demonstrated a similar tendency to HAT inhibitory activities; EGCG $>$ EGC $>$ EC. The strongest HAT inhibitor among them, EGCG $(50 \mu \mathrm{M})$, downregulated AR acetylation and finally, AR protein translocation to nucleus from the cytoplasmic compartment was effectively inhibited in the presence of the agonist. These results suggest another mechanism to develop effective therapeutics based on green tea catechins.
\end{abstract}

\section{Introduction}

Catechins have been shown to have cancer chemopreventive effects against several types of cancer, including prostate cancer $(1,2)$. Like epigallocatechin-3-gallate (EGCG), other major catechins, epicatechin (EC) and epigallocatechin (EGC),

Correspondence to: Dr Ho-Geun Yoon, Department of Biochemistry and Molecular Biology, Center for Chronic Metabolic Disease Research, College of Medicine, Yonsei University, Seoul 120-752, Republic of Korea

E-mail: yhgeun@yuhs.ac

${ }^{*}$ Contributed equally

Key words: epigallocatechin-3-gallate, histone acetyltransferase, prostate cancer, androgen receptor, acetylation also suppress androgen-dependent cell growth (3); however, they do not induce effective suppression of androgen-independent cell growth. Recent epidemiological data have associated tea consumption with a reduced frequency of hormone related cancers, including prostate cancer, suggesting that green tea catechins may affect hormone receptors (4). Androgen receptor (AR) is a hormone nuclear receptor that mediates androgen action in prostate cells (5). AR plays a key role in prostate cancer cellular proliferation and normal prostate development induced by the androgen, dihydrotestosterone (6). It has recently become clear that human prostate cancer development involves post-transcriptional modifications, such as acetylation and phosphorylation of the androgen receptor (7). Histone acetyltransferase (HAT) is one of the histone modifier proteins that acetylates AR during prostate cancer cell growth (8). AR is acetylated by p300, P/CAF, and TIP60, leading to the recruitment of co-regulators to the basal transcription machinery of AR target genes and growth properties of the receptors, in cultured cells and in vivo (7).

We have previously reported that EGCG suppresses RelA acetylation through its potent anti-HAT activity (9). Here, we show that the major catechins of green tea suppress AR acetylation through their anti-HAT activities, reducing prostate cancer cell growth. We demonstrate that catechins regulate androgen-mediated transcription in an androgendependent prostate cancer cell line, LNCaP. We also suggest possible mechanisms for catechin-induced cell death in androgen-dependent prostate cancer cells, which may aid in the development of effective chemopreventive therapeutics.

\section{Materials and methods}

Cell culture and reagents. Human LNCaP cells were maintained in RPMI-1640 (Gibco-BRL, Grand Island, NY, USA) supplemented with $10 \%$ fetal bovine serum (Gibco-BRL). Cells in 6-well plates were supplemented with fresh medium $2 \mathrm{~h}$ before transfection with $1.5 \mu \mathrm{g}$ DNA, according to the manufacturer's protocol for effective transfection (Qiagen). 
After 2-3 h incubation, cells were treated with medium supplemented with charcoal-dextran-treated FBS, containing either ethanol or ligands. Cell lysates were prepared for luciferase assays according to the manufacturer's instructions (Promega, Madison, WI, USA). Relative luciferase activities were plotted using the activity of AR in the presence of the ligand, R1881 $(50 \mathrm{nM})$ and/or flutamide $(20 \mathrm{mM})$. The results were obtained from at least 3 sets of transfections and presented as mean values. Major catechins, EGCG, EC and EGC were purchased from Sigma-Aldrich (St. Louis, MO, USA). Antibodies against acetylated $\mathrm{H} 3$ and p300 were purchased from Upstate Biotechnology (Lake Placid, NY, USA), and AR and acetylated lysine residues were from Santa Cruz Biotechnology, Inc. (Santa Cruz, CA, USA), Lipofectamine 2000 transfection reagent was purchased from Invitrogen.

Cell viability test. $\mathrm{LNCaP}$ and $\mathrm{PC} 3$ cells $\left(1 \times 10^{4}\right.$ cells/well $)$ and HeLa cells $\left(5 \times 10^{3}\right.$ cells/well) were seeded in 96-well culture plates with RPMI-1640 containing 10\% FBS and incubated overnight. After 48 incubation with major catechins in serum-free medium, 3-(4,5-dimethylthiazol-2-yl)-2,5diphenyltetrazolium bromide (MTT) solution in PBS was added and the plates were incubated for $2 \mathrm{~h}$. Afterwards, the medium was removed and the formed blue formazan was solubilized with DMSO. Absorbance was measured at $570 \mathrm{~nm}$ with background subtraction at $630 \mathrm{~nm}$.

HAT, HDAC, HMT and SIRT1 activity assay. A HeLa cell nuclear extract (NE) was prepared as previously described (10). HAT activity and histone deacetylase (HDAC) activity assays were determined using a commercially available kit (Biovision Biotechnology, Mountain View, CA, USA) according to the manufacturer's instructions. Histone methyltransferase (HMT) activity assays were performed using the manufacturer's protocol as previously described (11). For the HMT assay, core histone and assay buffer were from the HMT assay reagent kit (Upstate Biotechnology). Silent mating type information regulation 2 homologue (Sirtruin; SIRT) 1 activity was assayed with the CycLex SIRT1/Sir2 Deacetylase Fluorometric Assay kit (CycLex, Nagano, Japan).

Dual-luciferase assay. LNCaP cells were seeded at $5 \times 10^{5}$ cells/ well in 6-well culture plates and incubated overnight with RPMI-1640 containing 10\% FBS. At 90\% confluence, $0.5 \mu \mathrm{g}$ of pGL3-PSA and $1 \mathrm{ng}$ of pGL-5 SV40 per 6 wells were transfected into LNCaP cells to perform a dual luciferase assay (Promega). The medium was replaced with phenol redfree RPMI-1640 media containing 5\% charcoal-stripped fetal bovine serum. After $48 \mathrm{~h}, 50 \mathrm{nM}$ of the synthetic androgen R1881 treatment, $20 \mu \mathrm{M}$ flutamide (Sigma-Aldrich) or EGCG $(50 \mu \mathrm{M})$ was added. Cell were lysed and analyzed after $18 \mathrm{~h}$ by MicroLumat Plus (EG\&G Berthold, Germany).

RNA extraction, real-time PCR analysis, and ChIP assays. RNA extraction, real-time PCR, and ChIP were performed, as described (12). The RT-PCR analysis and quantification was performed with SYBR-Green PCR Master Mix reagents on an ABI PRISM 7300 Sequence Detection System (Applied Biosystems, Foster City, CA, USA). The singularity and specificity of amplification were verified by a dissociation analysis software. All samples were normalized to human GAPDH. Primer sequences for amplification of NKX3-1 RNA were (F, 5'-CTGTCAGCCCCTGAACGG-3' and R, 5'-AACC ATATCTTCACTTGGGTCTCC-3'). Primer sequences for amplification of the PSA RNA were (F, 5'-AGTCTGAGGA GGTGTTCTGGTG-3' and R, 5'-GAGGTCGTGGCTGGA GTCATCA-3'). Primers used for ChIP analysis were: PSA (ARE1), 5'-TTGCATAAATTAGGGGAGAACATACCA-3' and 5'-GAGGGACCCAGCTGCGATTCA-3. All reactions were performed in triplicate. Relative expression levels and SDs were calculated using the comparative method.

Immunostaining. LNCaP cells were cultured in $2 \times 2-\mathrm{cm}^{2}$ Lab-Tek chamber slides and the medium was replaced with phenol-free RPMI-1640 containing 10\% charcoal-stripped FBS for 48 h. Cells were treated with EGCG and R1881 for $24 \mathrm{~h}$, and then fixed with $4 \%$ formaldehyde at room temperature for $10 \mathrm{~min}$. The fixed cells were rinsed with PBS and then stained with DAPI to detect nuclei and rhodamine-conjugated AR antibody. Cells were examined with the DeltaVision RT Imaging Microscope system (Applied Precision, Inc.).

\section{Results}

Major catechins induce anti-histone acetyltransferase activitydependent prostate cancer cell death.LNCaP,PC3 and HeLa cells were cultured with various concentrations of EC, EGC or EGCG for 2 days. As shown in Fig. 1A, EGCG, EGC and EC induced concentration-dependent cell death in the androgen-dependent prostate cancer, LNCaP cells, but in the androgen-independent prostate cancer cell, PC3, cell death by catechins did not dramatically increase compared to LNCaP cells (Fig. 1B). In HeLa cells, catechins did not affect cell viability in any concentrations (Fig. 1C). Since catechin derivatives have been known to possess anti-HAT activity (9), we next examined whether the differential effects of catechins on prostate cancer cell death is correlated with those of anti-HAT activities. Although EGCG, EGC and EC exhibited HAT inhibitory activities up to $70-90 \%$ at $100 \mu \mathrm{M}$, EGCG exhibited the highest anti-HAT activity among catechin derivatives (Fig. 2A). To assure that the inhibition of HAT activity was specific, we tested HDAC and HMT activities in cells treated with the same set of catechins. The results of these assays showed that none of the catechins affected HDAC or HMT activity (Fig. 2B and C). To further confirm the enzyme specificity of catechins, we performed SIRT deacetylase assays using HeLa nuclear extract. Similar to the results of the HDAC and HMT assays, the SIRT deacetylase assay showed no difference either in the presence or absence of catechins (Fig. 2D). Collectively, these results indicate that the selective suppression of androgen-dependent prostate cancer cell growth is positively correlated with the anti-HAT activities from catechins.

EGCG exhibits the highest inhibitory effects on androgenmediated transcription in LNCaP cells. To measure the effects of EC, EGC and EGCG on AR-mediated transcription in LNCaP cells, we used an androgen-dependent reporter vector (pGL3-PSA) containing the androgen responsive element and the luciferase reporter gene. LNCaP cells were treated with a synthetic androgen, R1881, in the presence or absence of EC, EGC or EGCG $(50 \mu \mathrm{M})$ for $48 \mathrm{~h}$ in RPMI-1640 containing 

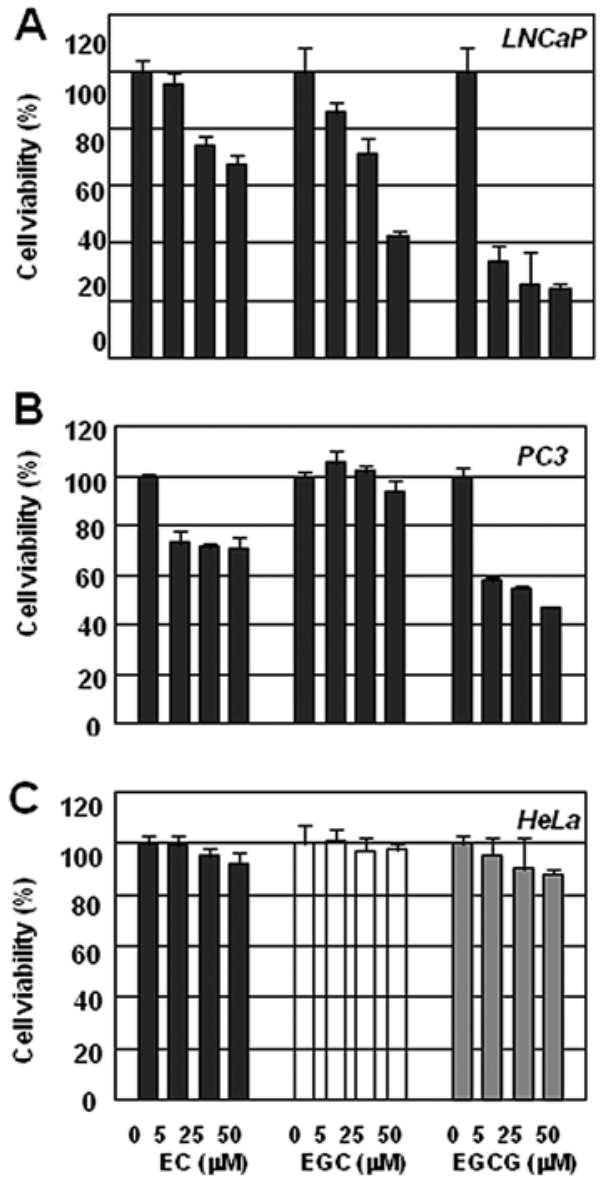

Figure 1. Effects of green tea catechins EC, EGC and EGCG on prostate cancer cells and HeLa cell. Cells were treated with serum-free medium containing $0,5,25$ or $50 \mu \mathrm{M}$ of EC, EGC or EGCG for $48 \mathrm{~h}$. (A) Results of the growth assay for LNCaP cells: EC, EGC and EGCG-induced cell death in the androgen-dependent prostate cancer cell line, LNCaP. (B) Results of the growth assays for PC3 cells: EC, EGC and EGCG had no significant effects on cell viability in the androgen-independent prostate cancer cell line, PC3. (C) Results of growth assays for HeLa cells: EC, EGC and EGCG had no significant effects on cell viability of HeLa cells. Results represent the mean value with SD from five independent experiments.

charcoal-stripped FBS. Incubation with R1881 resulted in a dramatic increase in luciferase activity, whereas co-treatment with EGCG resulted in an $80 \%$ decrease in luciferase activity (Fig. 3A). Consistent with the assays for cell death (Fig. 1A) and the specific anti-HAT activities of the individual catechins (Fig. 2A), EGCG had the highest inhibitory effects on R1881bound AR-mediated transcription, followed by EGC and EC $(\mathrm{EGCG}>\mathrm{EGC}>\mathrm{EC})$.

To determine whether EC, EGC and EGCG inhibition of AR-regulated gene expression is dose-dependent in LNCaP cells, the mRNA levels of endogeneous AR-regulated genes (PSA and NKX3.1) were measured by real-time PCR. LNCaP cells were exposed to 0,50 or $100 \mu \mathrm{M}$ of each catechin for $18 \mathrm{~h}$, and total-RNA was then isolated. As expected, both an androgen-dependent increase and a catechin-induced reduction were observed in the mRNA levels of PSA and NKX3.1 (Fig. 3B). EGCG treatment led to the most dramatic reduction of AR target gene expression at 50 and $100 \mu \mathrm{M}$. EGC and EC treatments led to significant reductions at $100 \mu \mathrm{M}$, but the effect was relatively weak compared to EGCG treatment.

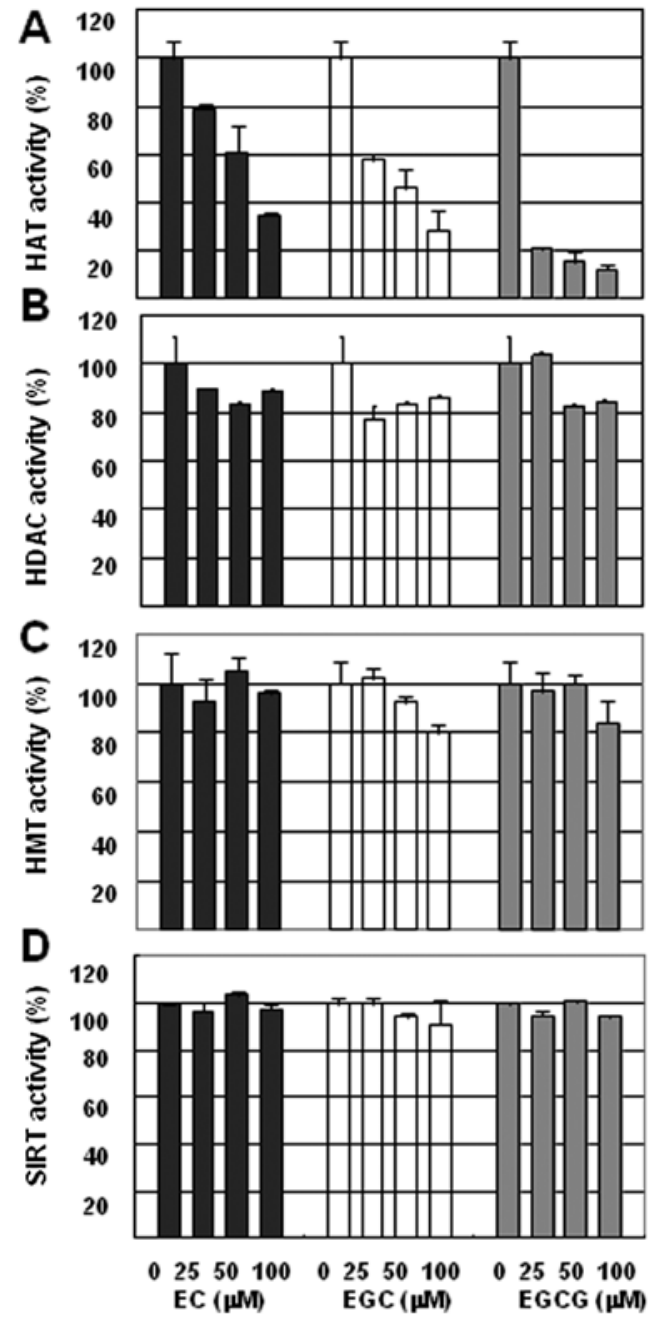

Figure 2. Effects of green tea catechins on HAT, HDAC, HMTase and SIRT activities. (A) HAT activity assays were performed with HeLa cell extracts treated with catechins (EC, EGC and EGCG). (B) HDAC activity assays were performed with HeLa cell extracts treated with catechins (EC, EGC and EGCG). (C) In vitro HMTase activity was assessed after treatment with catechins. (D) SIRT1 activity was assayed with SIRT1/sir2 Deacetylase Fluorometric Assay kit. Deacetylase enzyme activity was assessed by measuring the fluorescence intensity. Results represent the mean value with SD from three experiments.

Together, these studies indicate that EGCG exhibits the highest inhibitory effect on AR-mediated transcription and induction of prostate cancer cell death.

EGCG suppresses the p300-mediated histone and AR acetylation in LNCaP cells. To confirm the relationship between anti-HAT activity and prostate cancer suppression, we measured the AR acetylation and the histone acetylation status of chromatin before and after treatment with EGCG. LNCaP cells were treated with R1881 or EGCG $(50 \mu \mathrm{M})$, either individually or in combination for $24 \mathrm{~h}$. First, to determine whether AR acetylation is affected by EGCG treatment in vivo, immunoprecipitation was performed on $\mathrm{LNCaP}$ cell lysate with a specific AR antibody and western blot analysis was subsequently used to determine the amount of acetylation at specific lysine residues. AR acetylation was increased in LNCaP cells in the presence of R1881, and this acetylation was decreased by EGCG treatment (Fig. 4A). We then 
A
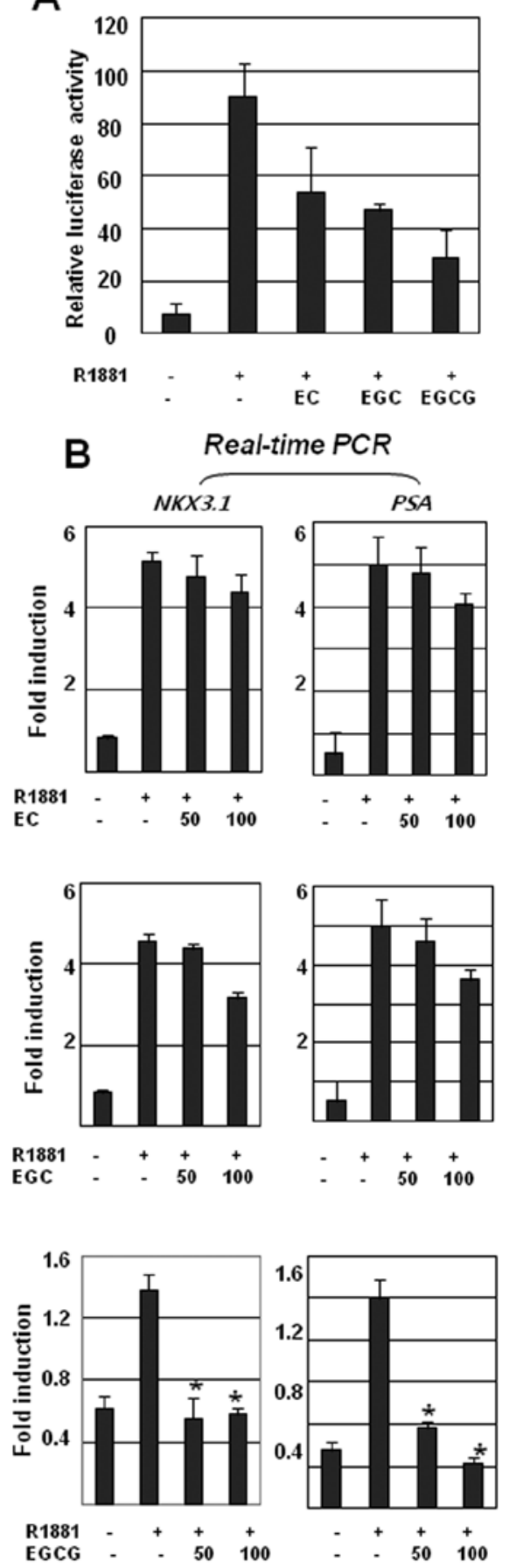

Figure 3. Effects of catechins on AR-mediacted transcription. (A) LNCaP cells were transiently transfected with pGL3-PSA and pGL5 SV40. Cells were treated with $50 \mu \mathrm{M}$ of EC, EGC or EGCG in the presence of $50 \mu \mathrm{M} \mathrm{R} 1881$ for $24 \mathrm{~h}$. (B) Real-time PCR of NKX3.1 and PSA were performed $24 \mathrm{~h}$ after treatment with 0,50 or $100 \mu \mathrm{M}$ of EC, EGC or EGCG in the presence or absence of R1881. Data are expressed as the mean \pm SD of three independent experiments, and presented as fold induction. "Indicates significant difference vs. the R-1881-treated group $(\mathrm{p}<0.05)$.

performed ChIP assays to observe histone acetylation and recruitment of p300 to the promoter region of the PSA gene. The R1881 treatment stimulated histone $\mathrm{H} 3$ acetylation and recruitment of p300 to PSA, however, EGCG suppressed the agonist-mediated recruitment of p300 to the promoter region (Fig. 4B). Collectively, these results demonstrated that EGCG reduced the acetylation of $\mathrm{AR}$ and histones.

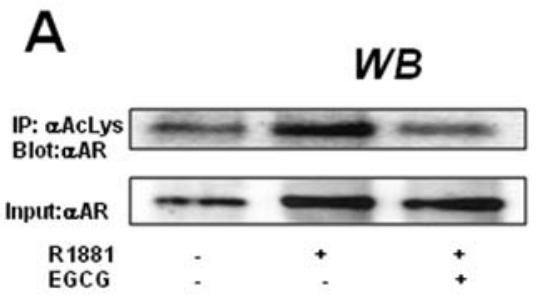

B

\section{ChIPassay}
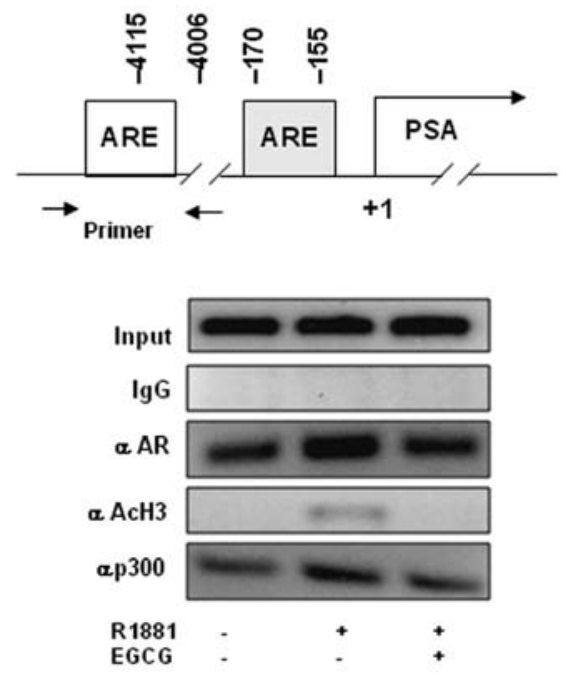

Figure 4. EGCG reduces the acetylation of AR and histones. (A) In vivo acetylation of the androgen receptor in LNCaP cells: cells were treated with $50 \mu \mathrm{M}$ EGCG in the presence or absence of R1881 for $24 \mathrm{~h}$. Cell lysates were immunoprecipitated with anti-Ac-lysine and western blot analyses were performed with anti-AR. (B) EGCG represses histone acetylation and dissociation of P300 from the AR response gene promoter region. Cells were treated with $50 \mu \mathrm{M}$ EGCG in the presence or absence of R1881 for $24 \mathrm{~h}$, then harvested, and the ChIP assay performed with anti-AR, anti-AcH3 and anti-p300.

EGCG inhibits the p300-enhanced hormone responsiveness of $A R$ and cellular proliferation. Since the overexpression of coactivators including p300 has been known to decrease the antagonist activities of flutamide and enhance the hormone responsiveness of $\mathrm{AR}$, we next investigated the effect of EGCG treatment on antagonist-bound AR-mediated transcription. For this experiment, the transcription reporter plasmids, pSG5-p300 and pGL3-PSA, were transiently transfected into LNCaP cells (Fig. 5A). As a positive control, AR activation by R1881 was abrogated by co-treatment with an androgen receptor inhibitor, flutamide $(20 \mu \mathrm{M})$. Expression of p300 in LNCaP cells resulted in a 1.5-fold increase in luciferase activity with R1881 treatment compared to LNCaP cells with R1881 alone. EGCG treatment $(50 \mu \mathrm{M})$ repressed transcription of the PSA reporter gene and prevented hyperacetylation of the AR receptor by p300 to a level similar to flutamide treatment, suggesting that EGCG inhibited p300-mediated PSA transcription. In the presence of ligand, AR translocates to the nucleus to access its target genes (13). Therefore, to examine whether agonist-induced AR acetylation is a critical step for nuclear translocation of the AR, we next investigated the subcellular localization of AR with or without EGCG in 
A

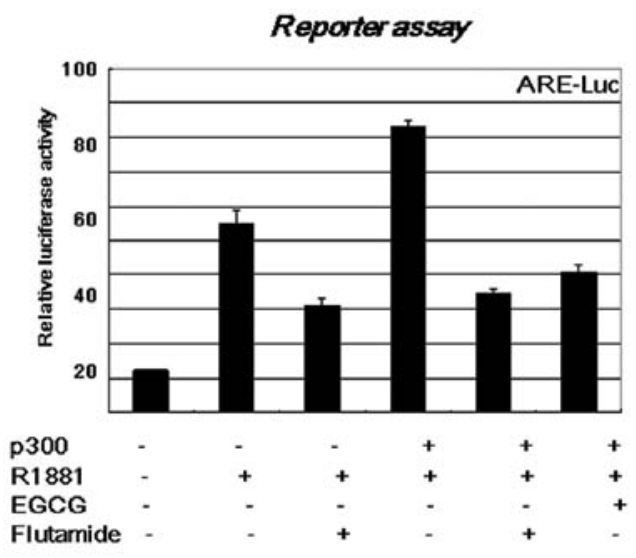

B

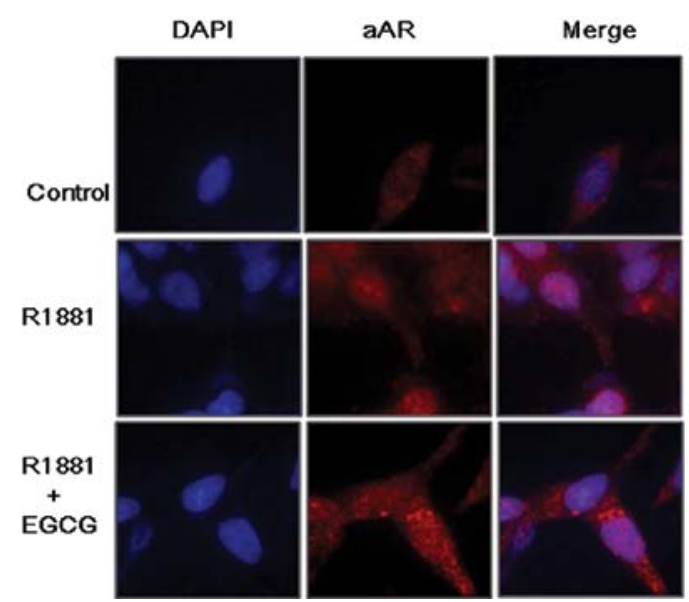

Figure 5. EGCG inhibits p300-mediated PSA transcription and agonist-dependent nuclear translocation of the androgen receptor. (A) EGCG inhibits p300-mediated PSA transcription. LNCaP cells were transiently transfected with pGL3-PSA only or pGL3-PSA plus pSG5-p300. pGL5-SV40 was used for normalization. Cells were treated with control, R1881, R1881/1 $\mu \mathrm{M}$ flutamide, or R1881/1 $\mu \mathrm{M}$ flutamide/50 $\mu \mathrm{M}$ EGCG for $24 \mathrm{~h}$. (B) EGCG inhibits androgendependent nuclear translocation of AR. LNCaP cells were treated with $50 \mu \mathrm{M}$ EGCG for $12 \mathrm{~h}$ and R1881 was then added. After $8 \mathrm{~h}$, fixation and chemical treatments were performed with rhodamine-conjugated AR antibody to detect AR localization and DAPI for the detection of nuclei.

LNCaP cells. For this experiment, LNCaP cells were treated with or without EGCG $(50 \mu \mathrm{M})$ in the presence of $50 \mathrm{nM} \mathrm{R} 1881$ for $18 \mathrm{~h}$. In the absence of R1881, AR was distributed in the cytoplasm and nucleus; however, AR protein was localized in the nucleus with R1881 treatment (Fig. 5B). Co-treatment with EGCG and R1881 resulted in the inhibition of AR translocation and the retention of AR in the cytoplasm. Thus, EGCG inhibits acetylation-dependent AR translocation, which leads to prostate cancer cell death, particularly in androgen-dependent cells. These results reveal a mechanism by which EGCG, and likely EC and EGC, induces prostate cancer cell death via inhibition of agonist-induced AR translocation through its anti-HAT activity.

\section{Discussion}

Several HAT inhibitors derived from natural products $(14,15)$ have been discovered and found to have potent chemopreventive and therapeutic activities against a wide variety of tumors. Nevertheless, compared to HDAC inhibitors, the antitumor effects of HAT inhibitors have been less well-characterized. We have previously reported that EGCG is an effective HAT inhibitor (9), which inhibits the acetylation of the RelA (p65) subunit of nuclear factor $-\kappa \mathrm{B}(\mathrm{NF}-\kappa \mathrm{B})$. Here, we suggest that the green tea catechins EGCG, EGC and EC induce androgen-dependent prostate cancer cell death by inhibiting AR-mediated transcription through their anti-HAT activities.

First, we demonstrated that the relative efficacy of individual green tea catechins in promoting androgen-dependent prostate cancer cell death varies directly with the strength of anti-HAT activity (EGCG $>$ EGC $>$ EC). Interestingly, activity differences among the individual catechins were also detected in reporter assays and measurements of AR-target gene expression. EGCG is one of the most promising growth suppressors for a variety of tumors, including prostate cancer, as has been suggested by numerous researchers $(16,17)$. Here, we demon- strate that EGCG can effectively suppress androgen-dependent prostate cancer cell growth and proliferation. Transcription factors, such as AR and ER $\alpha$, are regulated through acetylation by HATs $(7,8)$ and there is strong evidence that AR acetylation is involved in the regulation of prostate cell growth, apoptosis and proliferation (13). Therefore, we examined whether the growth inhibitory effects of EC, EGC and EGCG on androgen-dependent prostate cancer cells are dependent on their anti-HAT activities. We demonstrated that the increased AR acetylation, histone $\mathrm{H} 3$ acetylation, and p300 recruitment induced by androgen was significantly suppressed by EGCG treatment. These results were confirmed by transcriptional reporter assays in LNCaP cells expressing the p300. EGCG was able to reduce gene transcription associated with p300 HAT activity to levels comparable to flutamide treatment. This result has a biological significance, because AR antagonists such as flutamide and bicalutamide are broadly used in the treatment of prostate cancer (18). The functional dependence of flutamide on prostate cancer has been demonstrated in several studies implies that the levels of HDAC or HAT in patients may be a determinant for the efficacy of anti-hormone therapy (19). Also, EGCG inhibits acetylation-dependent AR translocation in androgen-dependent cells. HAT protein promotes prostate cancer cell proliferation by translocation of AR into the nucleus (20), and EGCG may suppress prostate cancer by inhibiting this translocation. Siddiqui et al (21) reported that green tea EGCG blunts AR function in prostate cancer. Here, we suggest that EGCG may suppress tanslocation of AR into the nucleus by modulating acetyltion.

In addition, developing drugs with increased capability to inhibit the AR-p300 interaction is likely to improve the efficacy of current anti-hormone therapy. In conclusion, EGCG treatment effectively inhibited agonist-induced AR acetylation, and subsequently, reduced the hormone responsiveness of AR. Therefore, we suggest that the reduction of AR acetylation mediated by EGCG represses androgen signaling and 
thereby acetylation-dependent prostate cancer cell growth and proliferation. These observations will be useful in the design of clinical trials for HATi-based therapies against prostate cancer.

\section{Acknowledgements}

This study was supported by a grant of the Korean Health Technology R\&D Project, Ministry of Health \& Welfare, Republic of Korea (A100011).

\section{References}

1. Scaltriti M, Belloni L, Caporali A, Davalli P, Remondini D, Rizzi F, Astancolle S, Corti A and Bettuzzi S: Molecular classification of green tea catechin-sensitive and green tea catechin-resistant prostate cancer in the TRAMP mice model by quantitative real-time PCR gene profiling. Carcinogenesis 27 : 1047-1053, 2006.

2. Won SM, Park YH, Kim HJ, Park KM and Lee WJ: Catechins inhibit angiotensin II-induced vascular smooth muscle cell proliferation via mitogen-activated protein kinase pathway. Exp Mol Med 38: 525-534, 2006.

3. Stuart EC, Scandlyn MJ and Rosengren RJ: Role of epigallocatechin gallate (EGCG) in the treatment of breast and prostate cancer. Life Sci 79: 2329-2336, 2006.

4. Albrecht DS, Clubbs EA, Ferruzzi M and Bomser JA: Epigallocatechin-3-gallate (EGCG) inhibits PC-3 prostate cancer cell proliferation via MEK-independent ERK1/2 activation. Chem Biol Interact 171: 89-95, 2008.

5. Sun A, Tang J, Hong Y, Song J, Terranova PF, Thrasher JB, Svojanovsky S, Wang HG and Li B: Androgen receptor-dependent regulation of $\mathrm{Bcl}-\mathrm{xL}$ expression: Implication in prostate cancer progression. Prostate 68: 453-461, 2008.

6. Hara T, Miyazaki H, Lee A, Tran CP and Reiter RE: Androgen receptor and invasion in prostate cancer. Cancer Res 68: 1128-1135, 2008.

7. Gong J, Zhu J, Goodman OB Jr, Pestell RG, Schlegel PN, Nanus DM and Shen R: Activation of p300 histone acetyltransferase activity and acetylation of the androgen receptor by bombesin in prostate cancer cells. Oncogene 25: 2011-2021, 2006.

8. Faus $\mathrm{H}$ and Haendler B: Androgen receptor acetylation sites differentially regulate gene control. J Cell Biochem 104: 511-524, 2008.

9. Choi KC, Jung MG, Lee YH, Yoon JC, Kwon SH, Kang HB, Kim MJ, Cha JH, Kim YJ, Jun WJ, et al: Epigallocatechin3-gallate, a histone acetyltransferase inhibitor, inhibits EBV-induced B lymphocyte transformation via suppression of RelA acetylation. Cancer Res 69: 583-592, 2009.
10. Choi HK, Choi KC, Oh SY, Kang HB, Lee YH, Haam S, Ahn YH, Kim KS, Kim K and Yoon HG: The functional role of the CARM1-SNF5 complex and its associated HMT activity in transcriptional activation by thyroid hormone receptor. Exp Mol Med 39: 544-555, 2007.

11. Choi KC, Park S, Lim BJ, Seong AR, Lee YH, Shiota M, Yokomizo A, Naito S, Na Y and Yoon HG: Procyanidin B3, an inhibitor of histone acetyltransferase, enhances the action of antagonist for prostate cancer cells via inhibition of p300dependent acetylation of androgen receptor. Biochem $\mathrm{J} 433$ : 235-244, 2011

12. Lee YH, Jung MG, Kang HB, Choi KC, Haam S, Jun W, Kim YJ, Cho HY and Yoon HG: Effect of anti-histone acetyltransferase activity from Rosa rugosa Thunb. (Rosaceae) extracts on androgen receptor-mediated transcriptional regulation. J Ethnopharmacol 118: 412-417, 2008.

13. Fu M, Rao M, Wang C, Sakamaki T, Wang J, Di Vizio D, Zhang X, Albanese C, Balk S, Chang C, et al: Acetylation of androgen receptor enhances coactivator binding and promotes prostate cancer cell growth. Mol Cell Biol 23: 8563-8575, 2003.

14. Kaulfuss S, Grzmil M, Hemmerlein B, Thelen P, Schweyer S, Neesen J, Bubendorf L, Glass AG, Jarry H, Auber B and Burfeind P: Leupaxin, a novel coactivator of the androgen receptor, is expressed in prostate cancer and plays a role in adhesion and invasion of prostate carcinoma cells. Mol Endocrinol 22: 1606$1621,2008$.

15. Balasubramanyam K, Varier RA, Altaf M, Swaminathan V, Siddappa NB, Ranga U and Kundu TK: Curcumin, a novel p300/ CREB-binding protein-specific inhibitor of acetyltransferase, represses the acetylation of histone/nonhistone proteins and histone acetyltransferase-dependent chromatin transcription. J Biol Chem 279: 51163-51171, 2004.

16. Sun Y, Jiang X, Chen S and Price BD: Inhibition of histone acetyltransferase activity by anacardic acid sensitizes tumor cells to ionizing radiation. FEBS Lett 580: 4353-4356, 2006.

17. Wang YC and Bachrach U: The specific anti-cancer activity of green tea (-)-epigallocatechin-3-gallate (EGCG). Amino Acids 22: $131-143,2002$

18. Hwang JT, Ha J, Park IJ, Lee SK, Baik HW, Kim YM and Park OJ: Apoptotic effect of EGCG in HT-29 colon cancer cells via AMPK signal pathway. Cancer Lett 247: 115-121, 2007.

19. Zhigang $Z$ and Wenlu S: Flutamide reduced prostate cancer development and prostate stem cell antigen mRNA expression in high grade prostatic intraepithelial neoplasia. Int J Cancer 122: 864-870, 2008

20. Yoon HG and Wong J: The corepressors silencing mediator of retinoid and thyroid hormone receptor and nuclear receptor corepressor are involved in agonist- and antagonist-regulated transcription by androgen receptor. Mol Endocrinol 20: 1048-1060, 2006.

21. Siddiqui IA, Asim M, Hafeez BB, Adhami VM, Tarapore RS and Mukhtar H: Green tea polyphenol EGCG blunts androgen receptor function in prostate cancer. FASEB J 25: 1198-1207, 2011. 